\title{
Effect of Vanadium Doping on Microstructure and Dielectric Behavior of $\mathrm{CaCu}_{3} \mathrm{Ti}_{4} \mathrm{O}_{12}$ Ceramics
}

\author{
Séka Simplice Kouassi ${ }^{1,}$, , Jean-Pierre Sagou Sagou ${ }^{2}$, Cécile Autret-Lambert ${ }^{3}$, Sonia Didry ${ }^{3}$, \\ Anoop Nautiyal ${ }^{3}$, Marc Lethiecq ${ }^{3}$ \\ ${ }^{1}$ Laboratory of Environmental and Materials Chemistry, Jean Lorougnon GUEDE University, Daloa, Ivory Coast \\ ${ }^{2}$ Laboratory of Materials Inorganics Chemistry, Félix Houphouët BOIGNY University, Abidjan, Ivory Coast \\ ${ }^{3}$ GREMAN UMR 7347 Laboratory, François Rabelais University, Tours, France
}

Email address:

sekasimplice@yahoo.fr (S. S. Kouassi)

${ }^{*}$ Corresponding author

\section{To cite this article:}

Séka Simplice Kouassi, Jean-Pierre Sagou Sagou, Cécile Autret-Lambert, Sonia Didry, Anoop Nautiyal, Marc Lethiecq. Effect of Vanadium Doping on Microstructure and Dielectric Behavior of $\mathrm{CaCu}_{3} \mathrm{Ti}_{4} \mathrm{O}_{12}$ Ceramics. International Journal of Materials Science and Applications. Vol. 6, No. 1, 2017, pp. 54-64. doi: 10.11648/j.ijmsa.20170601.18

Received: December 16, 2016; Accepted: December 27, 2016; Published: January 21, 2017

\begin{abstract}
In this work, effect of vanadium doping of $\mathrm{CaCu}_{3} \mathrm{Ti}_{4} \mathrm{O}_{12}$ (CCTO) on microstructure and complex dielectric constant over wide frequency $(100 \mathrm{~Hz}-1 \mathrm{MHz})$ and temperature $\left(0^{\circ} \mathrm{C}-160^{\circ} \mathrm{C}\right)$ ranges has been studied. The vanadium doping of CCTO system results in an increase of grain size, the grains being surrounded by melted-like grain boundaries. Real parts of dielectric constant of all samples are similar at low frequency $(<1 \mathrm{kHz})$. In doped samples, above $1 \mathrm{kHz}$, a relaxation appears which is evidenced by a drop of real part of permittivity and a peak of its imaginary part. This relaxation phenomenon is very significant at relatively low doping rates and then decreases again as vanadium content increases. AC conductivity behavior of vanadium-doped CCTO can be divided in three regions depending on conduction processes. The calculated activation energies were close to $0.46 \mathrm{eV}$.
\end{abstract}

Keywords: Ceramics, Solid State Reaction Method, Microstructure, Dielectric Properties, Electrical Conduction

\section{Introduction}

Research on new high permittivity materials for the enhancement of the performance of capacitors has led to the discovery of a new class of materials based on perovskiterelated compounds like the prominent $\mathrm{CaCu}_{3} \mathrm{Ti}_{4} \mathrm{O}_{12}$ (CCTO). This material shows a number of interesting properties with the possibility of a large number of technological applications in the field of microelectronics [1]. The principal application for such a dielectric ceramic is for capacitors in electronic circuits and as electrical insulators [2]. Indeed, it presents a high dielectric constant almost independent of frequency in a huge frequency range $\left(10^{2}-10^{6} \mathrm{~Hz}\right)$ as well as has a high thermal stability over a wide temperature range $(100-600 \mathrm{~K})$ [3, 4, 5]. However, the properties of bulk samples are not well understood and are the object of numerous discussions. Nevertheless, it seems more or less accepted that the origin of the high dielectric constant is due to extrinsic effects, like Surface Barrier Layer Capacitance (SBLC) [6] or Internal Barrier Layer Capacitance (IBLC) [1, $7,8]$. This last theory reported that semi-conductor grains and insulating grain boundaries lead to this large value of permittivity. It is well known that the dielectric properties of CCTO strongly depend on processing conditions during sample preparation, on oxygen stoichiometry, on sintering additive, as well as on small amounts of cationic doping [9, $10,11]$. In all cases, to improve the properties, the grains must be semi-conductor. Adams and al. proposed that the semi-conductivity could come from the generation of oxygen vacancies [12]. $\mathrm{Li}$ and al. suggested that $\mathrm{Cu}^{2+}$ reduces to $\mathrm{Cu}^{+}$ during the synthesis with a small substitution of $\mathrm{Ti}^{4+}$ on $\mathrm{Cu}$ sites. To compensate the charge balance, $\mathrm{Ti}^{3+}$ would appear within the material, thus affecting the conductivity [13].

For the present work, we directly added $\mathrm{V}_{2} \mathrm{O}_{3}$ powder with weight ratio of $0.5,1,3$ and $5 \%$ into CCTO powder and sintered these mixtures. We have investigated the structural, 
dielectric properties and conductivity as a function of $\mathrm{V}_{2} \mathrm{O}_{3}$ content.

\section{Experimental Procedure}

Single phase $\mathrm{CaCu}_{3} \mathrm{Ti}_{4} \mathrm{O}_{12}$ (CCTO) powders were prepared through a conventional solid state reaction route by mixing appropriate amounts of high purity $\mathrm{CaCO}_{3}(99.98 \%), \mathrm{TiO}_{2}$ $(99.9 \%)$, and $\mathrm{CuO}(99.99 \%)$ raw powders previously dried at $400^{\circ} \mathrm{C}$ for 24 hours. The starting materials were mixed and ball milled two days in isopropanol, followed by calcination at $950^{\circ} \mathrm{C}$ for $12 \mathrm{~h}$. The x-ray diffraction showed that the calcined powders were single phase. The calcined CCTO powders were then mixed with $0.5,1,3$ and $5 \%$ in mass of $\mathrm{V}_{2} \mathrm{O}_{3}$ powder and then compacted into pellets by cold isostatic pressing followed by sintering in air at $1050^{\circ} \mathrm{C}$ for $24 \mathrm{~h}$.
X-ray diffraction (XRD) analysis was performed (D8 diffractometer, Bruker) using $\mathrm{Cu} \mathrm{K}_{\alpha}$ radiation of wavelength $\approx 1.54 \AA$. The diffraction patterns were recorded in $2 \theta$ range of $10-110^{\circ}$ with a step of $0.02^{\circ}$. Rietveld refinement of the crystal structures was carried out with the FullProf software [14].

The morphology and microstructural analysis of the powders and pellets were performed by scanning electron microscopy (SEM) using a Hitachi 4160-F instrument.

Gold was deposited on both surfaces of the sintered disks and fired at $500^{\circ} \mathrm{C}$ for 1 hour. The sample pellets were 12 $\mathrm{mm}$ in diameter and about $1 \mathrm{~mm}$ in thickness. The dielectric properties and electric conductivity were measured over a broad frequency range $(100 \mathrm{~Hz}-10 \mathrm{MHz})$ using an Agilent 4294A impedance analyzer at various temperatures.

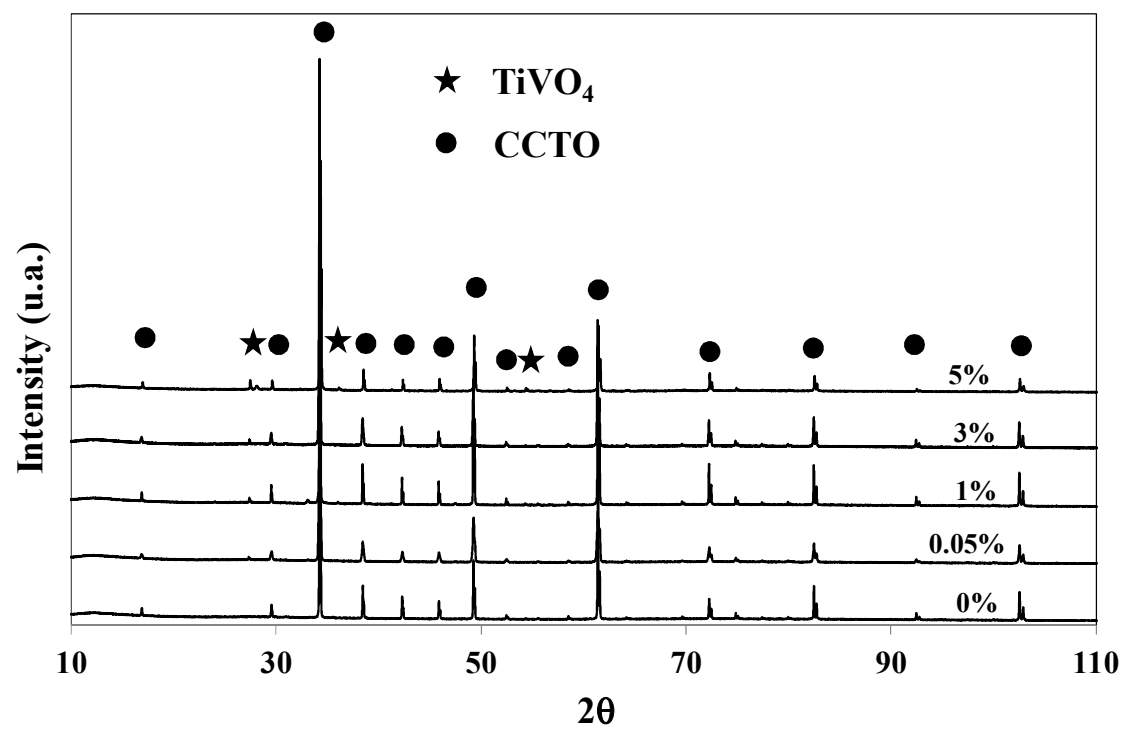

Figure 1. XRD patterns of pure and vanadium-doped CCTO samples.

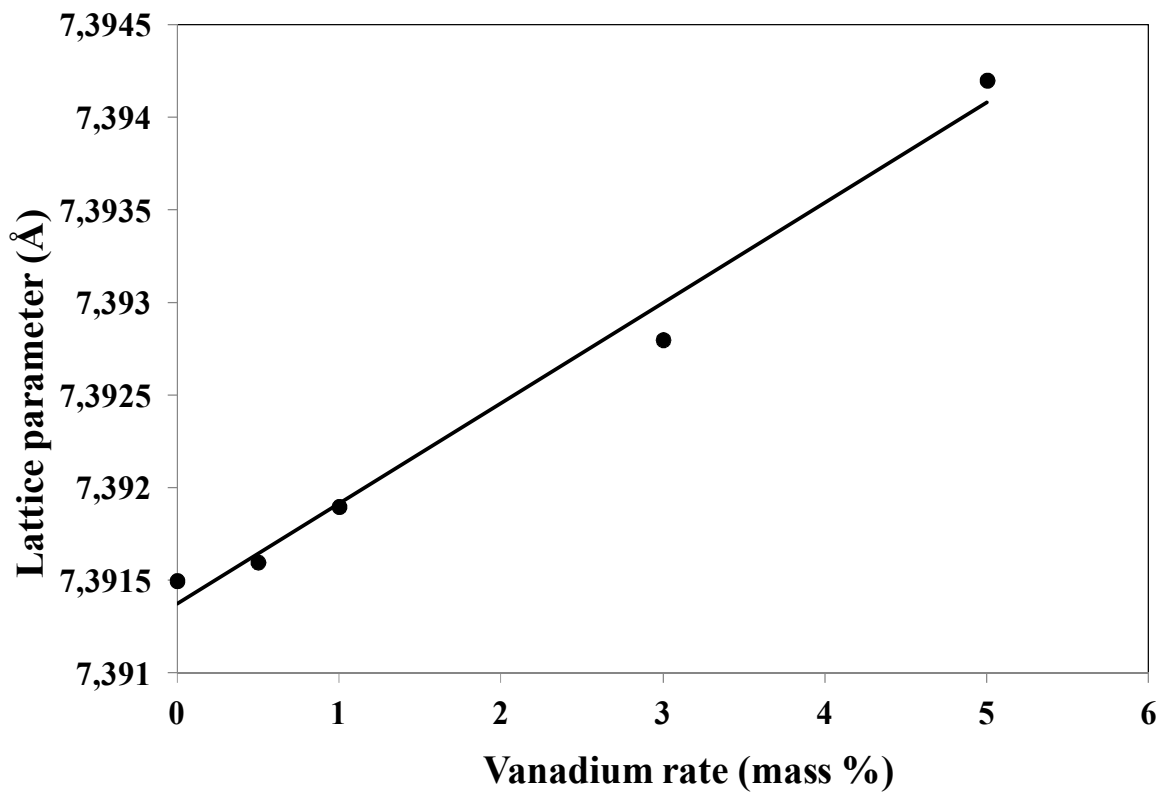

Figure 2. Lattice parameter depending on $V$ rate. 


\section{Results and Discussions}

\subsection{X-ray Analysis}

The X-ray diffraction (XRD) patterns of the samples are shown in Figure 1 for different $\mathrm{V}_{2} \mathrm{O}_{3}$ content. The peaks on diffraction patterns can be indexed to perovskite-related structure of CCTO with the cubic space group Im-3. However, impurities of $\mathrm{TiVO}_{4}$ are found in the $5 \%$ doped ceramics with a percentage of about 3-4\%, indicated by the symbol $\star$. Consequently, only samples with doping up to 3 mass $\%$ were considered for further analysis. $\mathrm{V}_{2} \mathrm{O}_{3}$ doping has a slight effect on the crystal structure of CCTO which is evidenced by an increase of the lattice parameter a of the cubic phase as doping increases (Figure 2). The evolution of a indicates that a part of Ti has been substituted by vanadium in the CCTO structure. Indeed, the volume evolution follows Vegard's law for the small doping. Taking account the radii and the valences of the cations, it seems that vanadium substitutes the octahedral Ti site.

\subsection{Microstructure Analysis}

Figures 3a, 3b, 3c, and 3d show the SEM images of the surface of $0,0.5,1,3$ mass $\% \mathrm{~V}_{2} \mathrm{O}_{3}$-added CCTO sample, respectively. In figure $3 \mathrm{a}$, one can observe that the pure CCTO compound has a microstructure consisting of closely linked grains with a size close to 3-4 $\mu \mathrm{m}$. The microstructure changes significantly with the addition of $\mathrm{V}_{2} \mathrm{O}_{3}$ (Figure 3). Figure $3 \mathrm{~b} \quad(0.5$ mass $\%)$ exhibits a non-homogeneous microstructure with large grains $(\approx 20 \mu \mathrm{m})$ surrounded by small ones $(5-10 \mu \mathrm{m})$. Figure $3 \mathrm{c}$ and $3 \mathrm{~d}$ ( 1 and 3 mass $\%)$ exhibit bigger grains than previously, surrounded by some melted-like grain boundaries.
It can be concluded that $\mathrm{V}_{2} \mathrm{O}_{3}$ doping increases the grain size of CCTO as evidenced by the microstructure shown in figure 3, these findings being similar to results obtained by Fang [15] and Brizé [9] with other dopants.
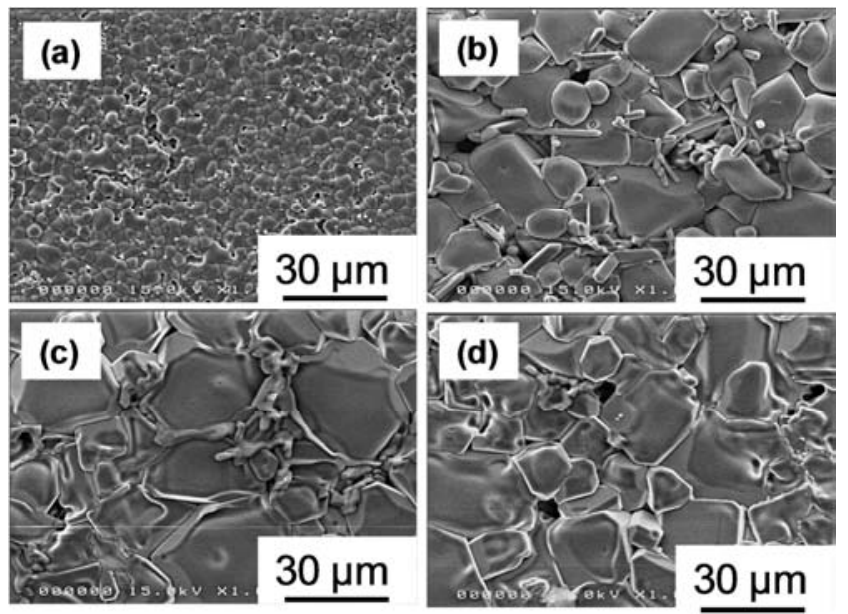

Figure 3. SEM micrographs of pure (a), $0.5 \mathrm{mass} \%$ (b), $1 \mathrm{mass} \%$ (c) and 3 mass\% (d) $\mathrm{V}_{2} \mathrm{O}_{3}$-doped-CCTO samples.

\subsection{Dielectric Properties}

Figure 4 shows the frequency variation of the real part of dielectric constant $\varepsilon$ ' of vanadium-doped CCTO ceramics: globally, a decrease is observed when frequency increases. Dielectric constant of pure and doped CCTO samples appear to be similar at low frequency $(<1 \mathrm{kHz})$ with a value over $10^{5}$ at room temperature, but it drops strongly over $1 \mathrm{kHz}$ for doped samples to reach approximately 100 .

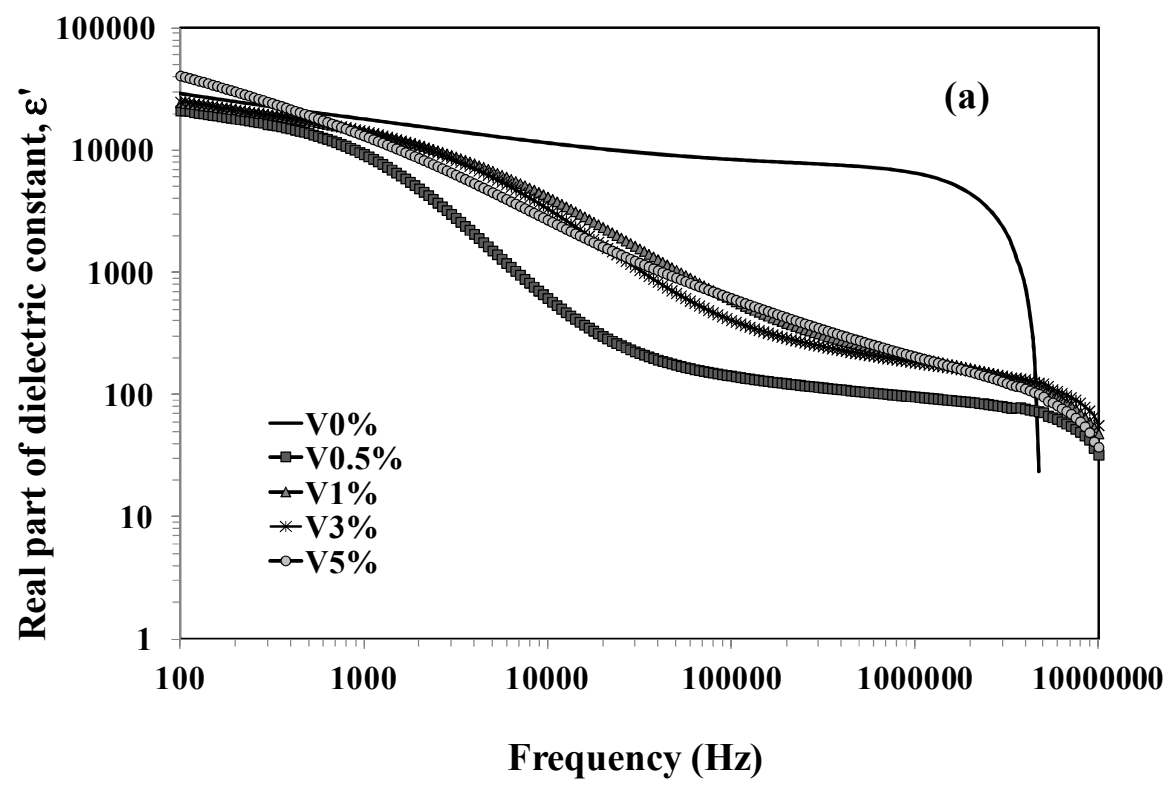

Figure 4. Variation of real part of dielectric constant with frequency for pure and vanadium-doped samples at room temperature.

The dependence of the complex dielectric constant with frequency reveals the existence of a relaxation process in the range $1 \mathrm{kHz}$ to $1 \mathrm{MHz}$ for doped samples, a phenomenon unobserved in pure CCTO. Un-doped CCTO exhibits a slightly decreasing 
real part of dielectric constant followed by a very strong decrease at high frequency [16]. The $\mathrm{V}_{2} \mathrm{O}_{3}$ doping also affects the loss tangent $\varepsilon " / \varepsilon$ (Figure 5), representing the AC conductivity, leading to its increase in the frequency range $1 \mathrm{kHz}-1 \mathrm{MHz}$ for all doped samples relatively to the un-doped one.

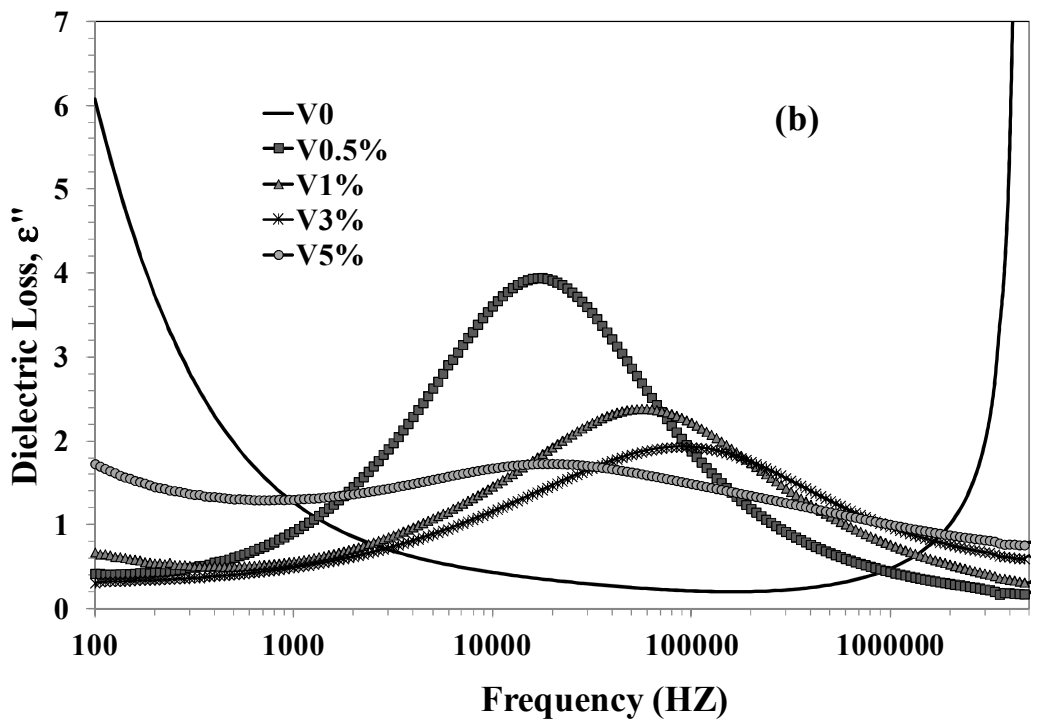

Figure 5. Frequency dependence of dielectric loss for pure and vanadium-doped CCTO samples at room temperature.

Real part of dielectric constant for pure CCTO shows weak temperature dependence [17]. With the addition of vanadium, a relaxor behavior corresponding to a decrease of $\varepsilon$ ' versus frequency associated with a peak in loss tangent becomes quite significant (Figure 6). Globally, in presence of vanadium, the magnitude of dielectric constant is decreased. The evolution of the real part of dielectric constant can be divided in two groups relative to the frequency. For frequencies up to $1 \mathrm{kHz}$, dielectric behavior is similar to that shown by pure CCTO on the entire temperature range. This behavior can be attributed to a Debye-like relaxation, generally associated to surface layers interfacial polarization. Similar results were reported by other authors [18, 19]. Recently, some authors [20] attributed this relaxation to a trap state related phenomenon: the high permittivity in the low frequency range of CCTO could be attributed to the trap states that exist at the electrode surface. Relaxation time is long enough to allow traps to respond, which could explain a huge permittivity at low frequency. When frequency is higher than $1 \mathrm{kHz}$, relaxor behavior is more pronounced for vanadium-doped CCTO, characterized by a decrease of the real part of dielectric constant on the entire temperature range. Vanadium-doped samples show a relaxation at higher frequencies and higher temperatures, which may be attributed to hopping of trapped carriers. The relaxation peak shifts towards higher temperatures when frequency increases. When vanaduim content increases, relaxation peaks are shifted towards lower temperatures.

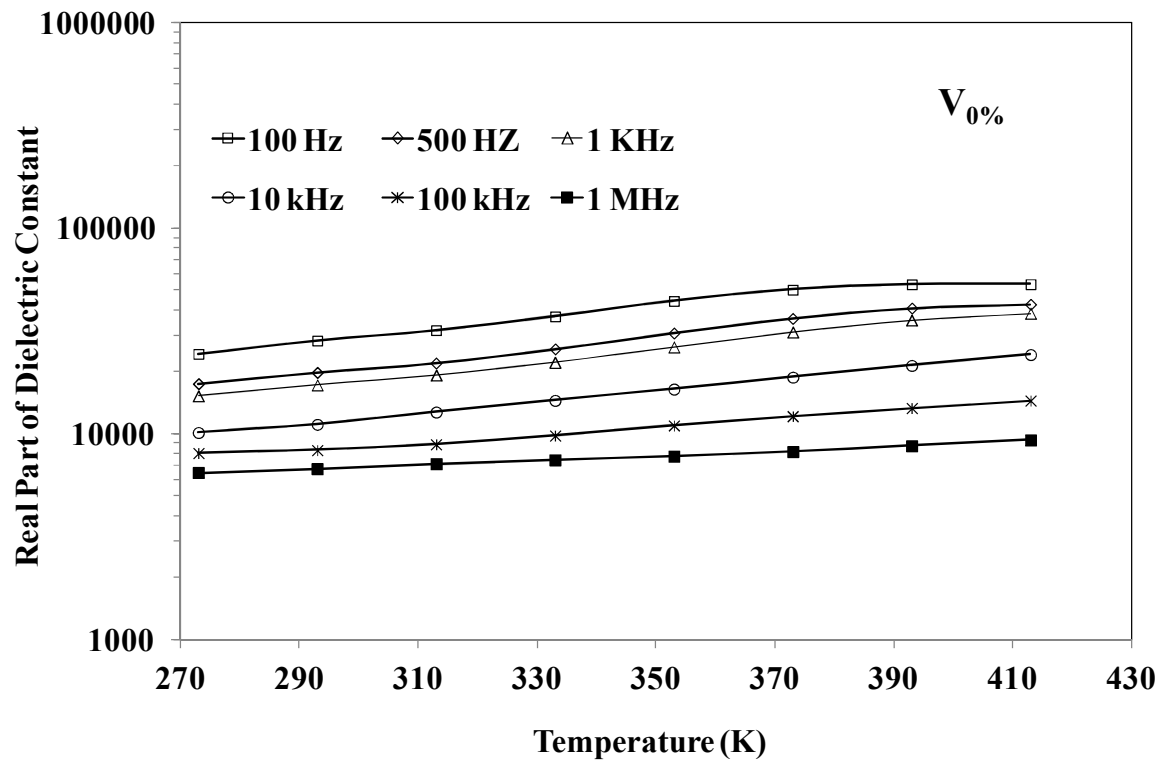



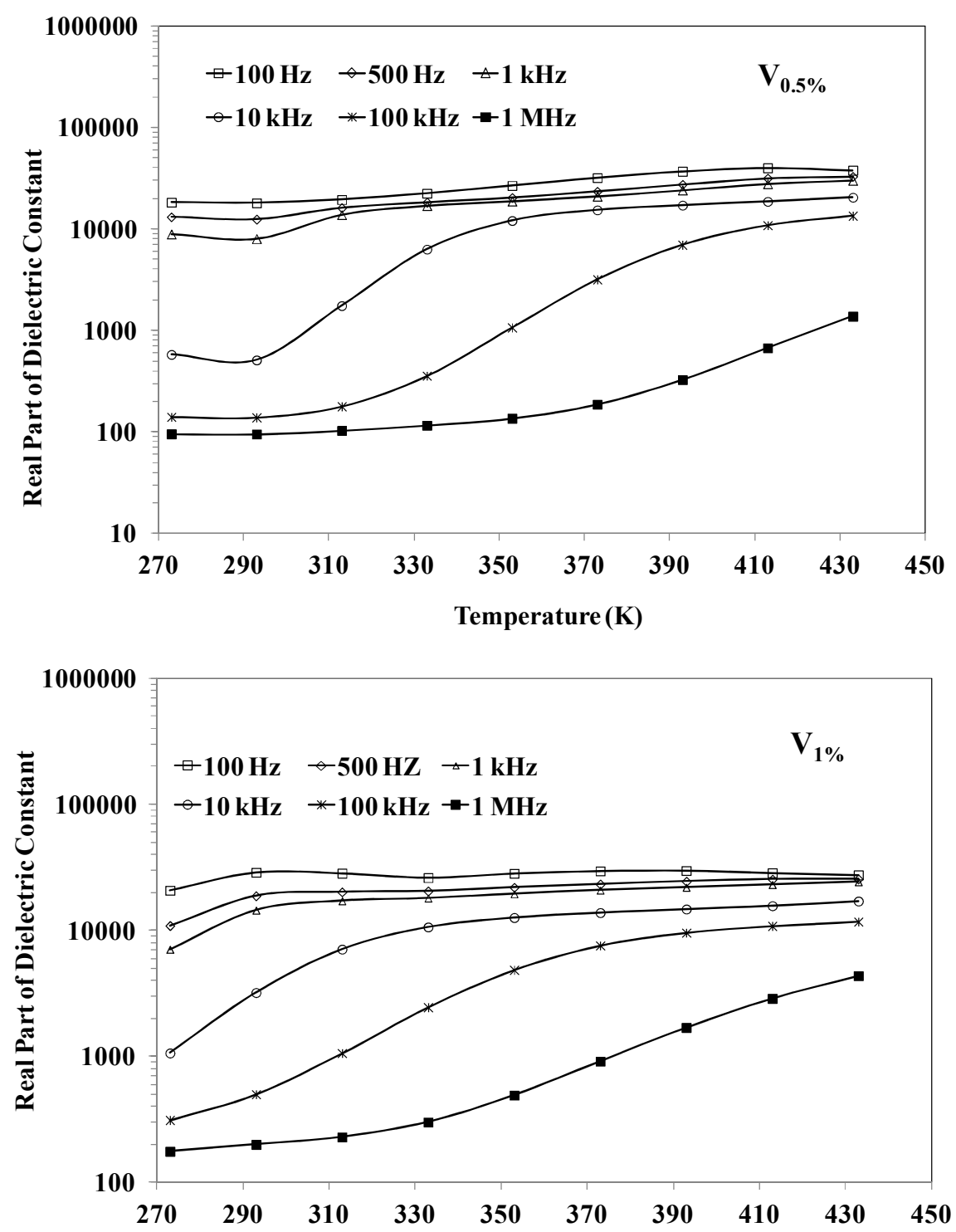

Temperature (K)

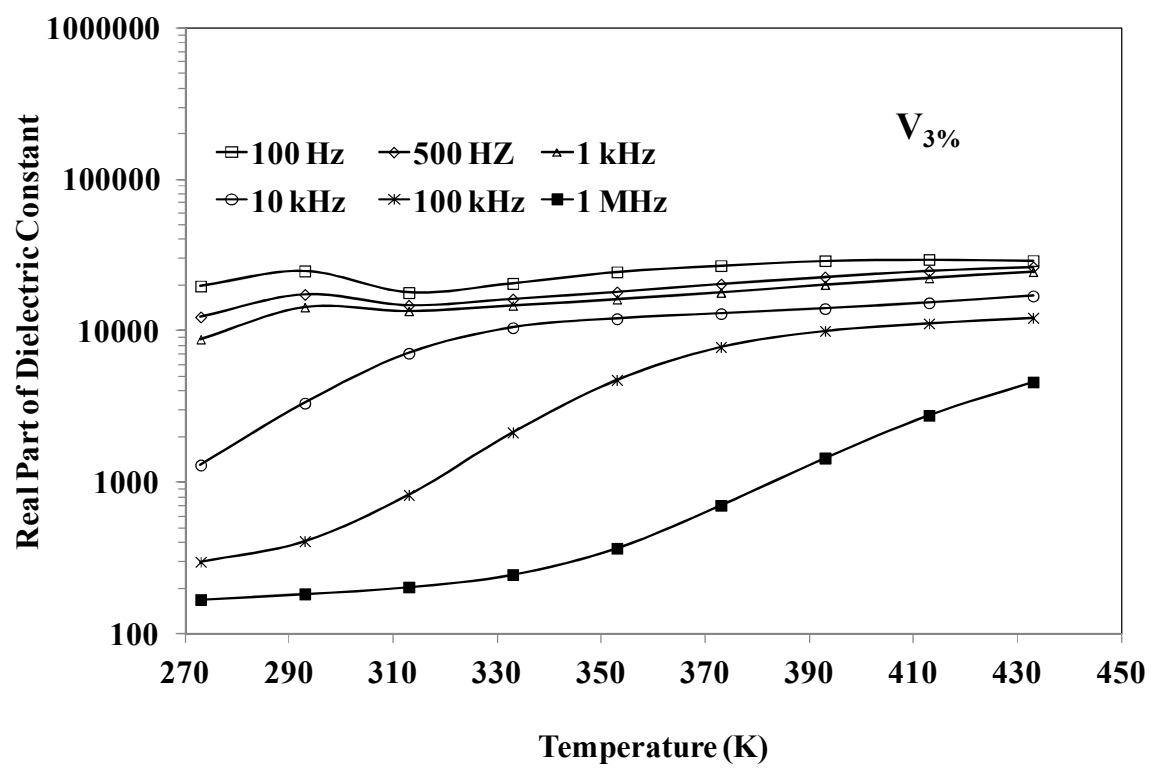

Figure 6. Variation of real part of dielectric constant $\varepsilon^{\prime}$ with temperature at different frequencies for pure and vanadium-doped CCTO samples. 
In Figure 7 it can be seen that dielectric losses increase as the temperature increases, indicating the semiconducting nature. For frequencies below $1 \mathrm{kHz}$, the dielectric losses decrease significantly in the presence of vanadium compared with the un-doped sample. Above $1 \mathrm{kHz}$, the dielectric loss is lower for pure CCTO, but peaks appear for doped compounds, indicating the relaxation phenomena mentioned above. The relaxation peak move to higher temperatures when the frequency increases and their amplitude decreases when the vanadium content increases.

Let us now consider the AC conductivity of different samples according to their $\mathrm{V}_{2} \mathrm{O}_{3}$ content. This conductivity results from hopping conduction and increases with frequency in disordered solids [21].

Equation (1) gives the expression of the total conductivity of a solid:

$$
\sigma=\sigma_{\mathrm{ac}}+\sigma_{\mathrm{dc}}
$$

with $\sigma_{\mathrm{ac}}$, the $\mathrm{AC}$ conductivity, originating from hopping conduction and $\sigma_{\mathrm{dc}}$ the DC conductivity resulting from conduction band.

Here, $\sigma_{\mathrm{ac}}$ was calculated using Equation 2:

$$
\sigma_{\mathrm{ac}}=2 \pi f \varepsilon_{0} \varepsilon^{\prime} \tan (\delta)
$$

where $f$ is the frequency, $\varepsilon_{0}$ the permittivity of vacuum, $\varepsilon^{\prime}$ the real part of dielectric constant and $\tan (\delta)$ the dielectric loss tangent. Figure 8 shows the frequency dependence of $\sigma_{\mathrm{ac}}$. The AC conductivity obeys the empirical formula of the frequency dependence given by the AC power law [15]

$$
\sigma_{\mathrm{ac}}=A f^{n}
$$

where $A$ and $n$ are constants, which depend on both the temperature and composition; $n$ is dimensionless constant having values between 0 and 1 .
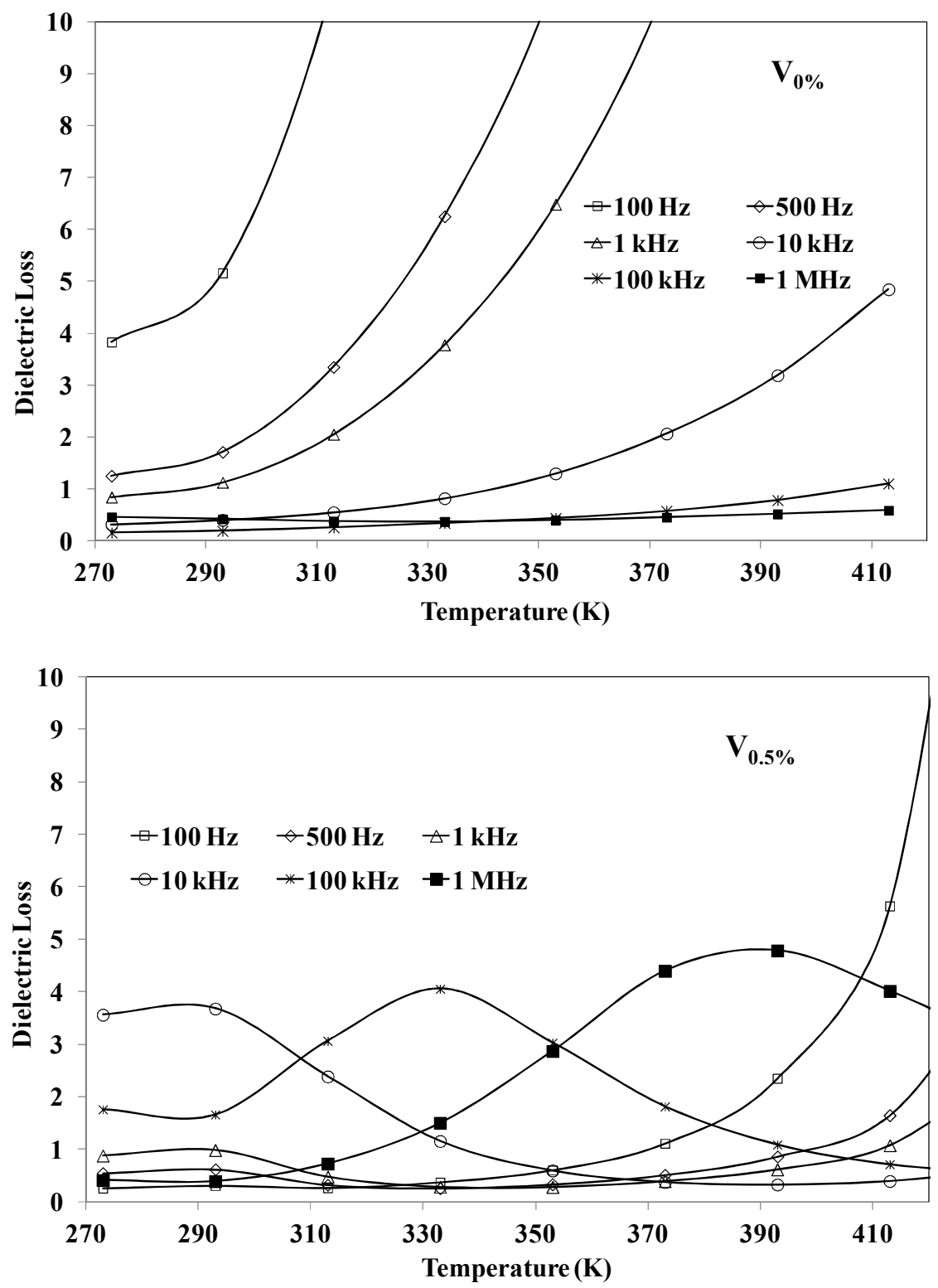

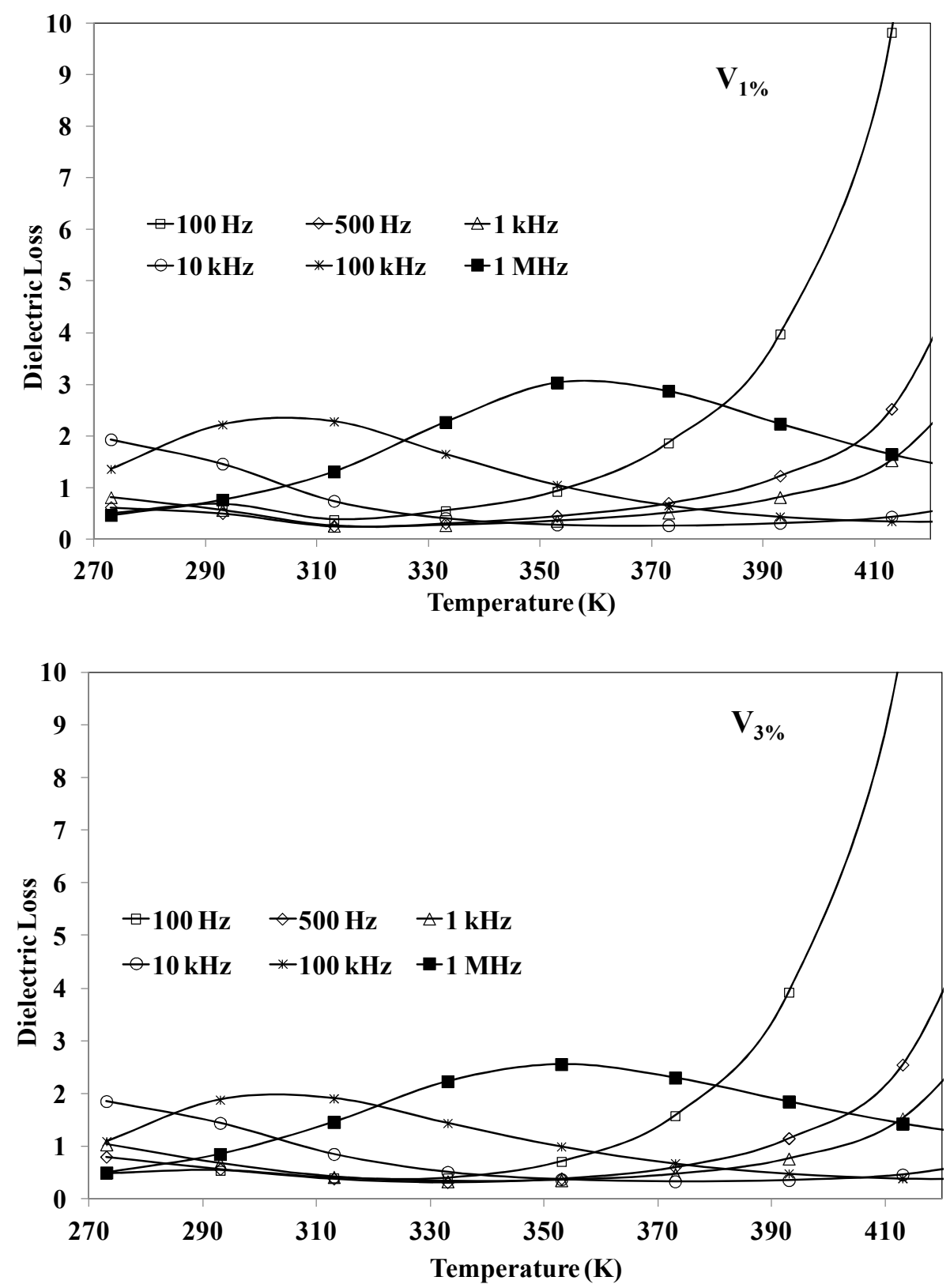

Figure 7. Variation of dielectric loss tangent with temperature at different frequencies for pure and vanadium-doped CCTO samples.

AC conductivity as a function of frequency can be divided into three regions: region I at low frequencies $(100 \mathrm{~Hz}-1$ $\mathrm{kHz})$, region II at intermediate frequencies $(1 \mathrm{kHz}-100$ $\mathrm{kHz}$ ) and region III at high frequencies (above $100 \mathrm{kHz}$ ). Let us first consider pure CCTO. In region I, the curve is practically constant and remains equal to the DC value, because the electric field does not influence the hopping conduction. In region II, the conductivity increases with frequency. This is due to the mechanism of hopping conduction that appears in region II and is enhanced in region III. For doped compounds, the AC conductivity starts at lower values, then increases strongly in region I and finally increases very slightly with frequency in regions II and III towards saturation. For the doped samples, the electric field under $1 \mathrm{KHz}$ is sufficient to influence the hopping conduction, which is not the case for pure CCTO. This result is in good agreement with that observed in Figure 4.

Properties of CCTO have been explained assuming it is made of semiconducting grains and insulating grain boundaries [7]. Here, it is first observed (Figure 8) that vanadium doping increases the resistivity of CCTO ceramics. $\mathrm{AC}$ conductivity is independent of frequency if a single charged species moves in an infinite lattice of identical potential wells. Secondly, the conductivity increases and saturates at high frequency when a single particle hopping to and from a double well with infinite height [22], which is also in favor of the assumption above. 


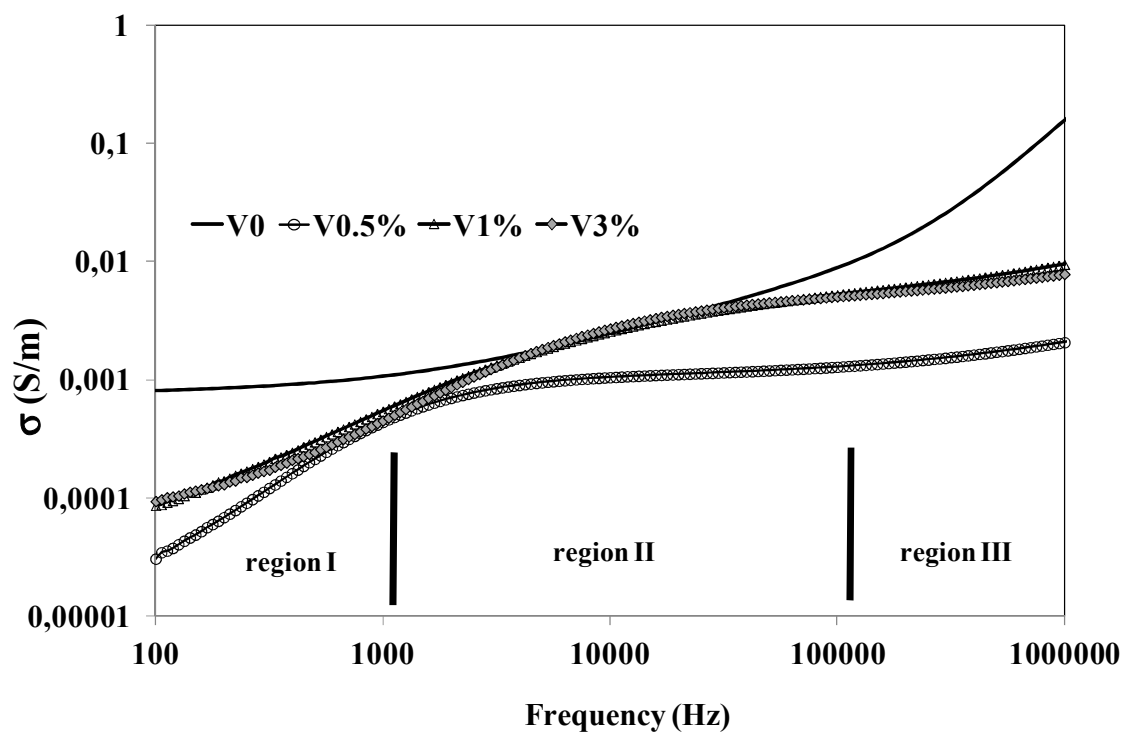

Figure 8. Variation of AC conductivity, $\sigma_{a c}$, with frequency at room temperature for pure and vanadium-doped CCTO samples.
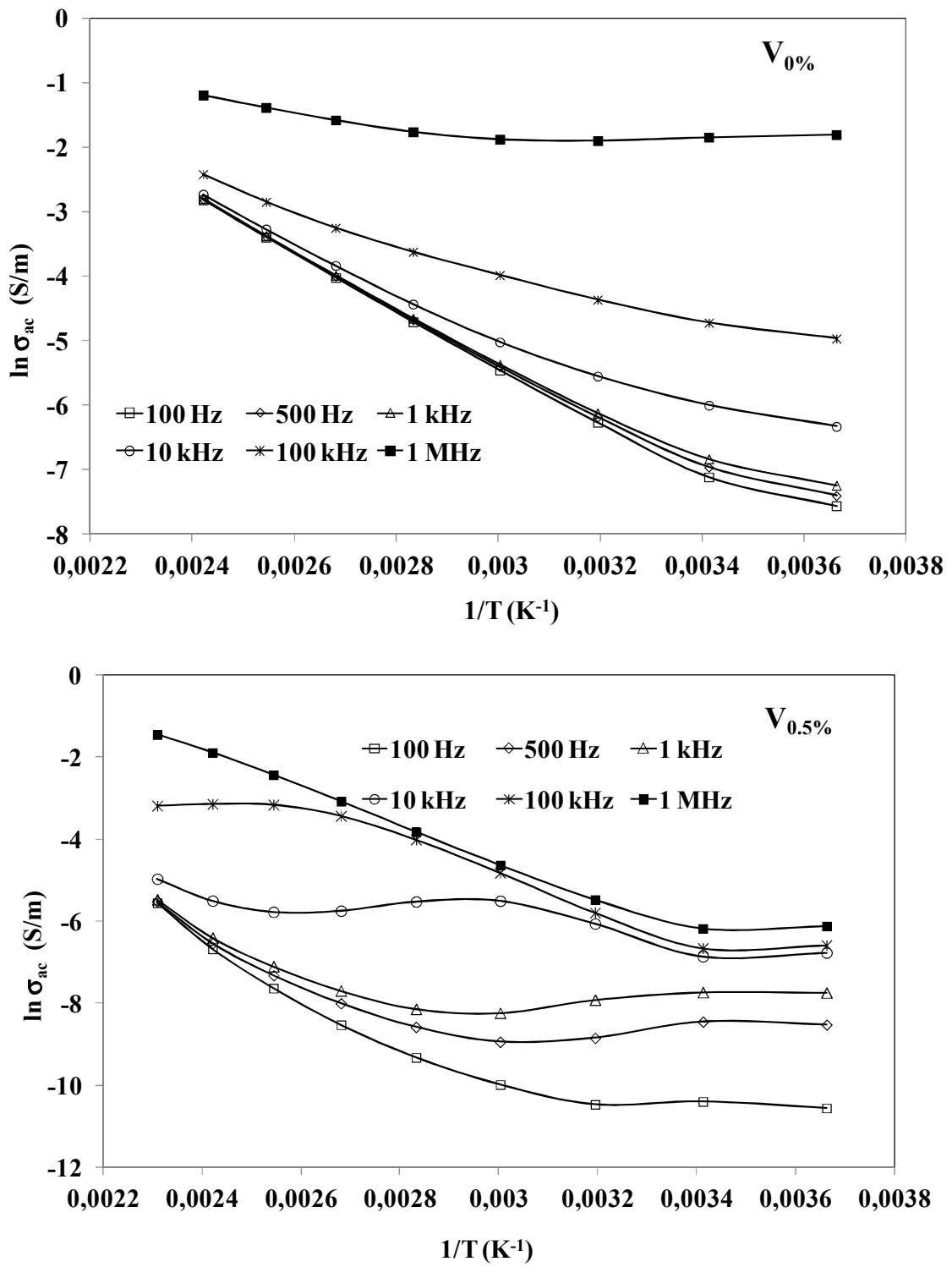

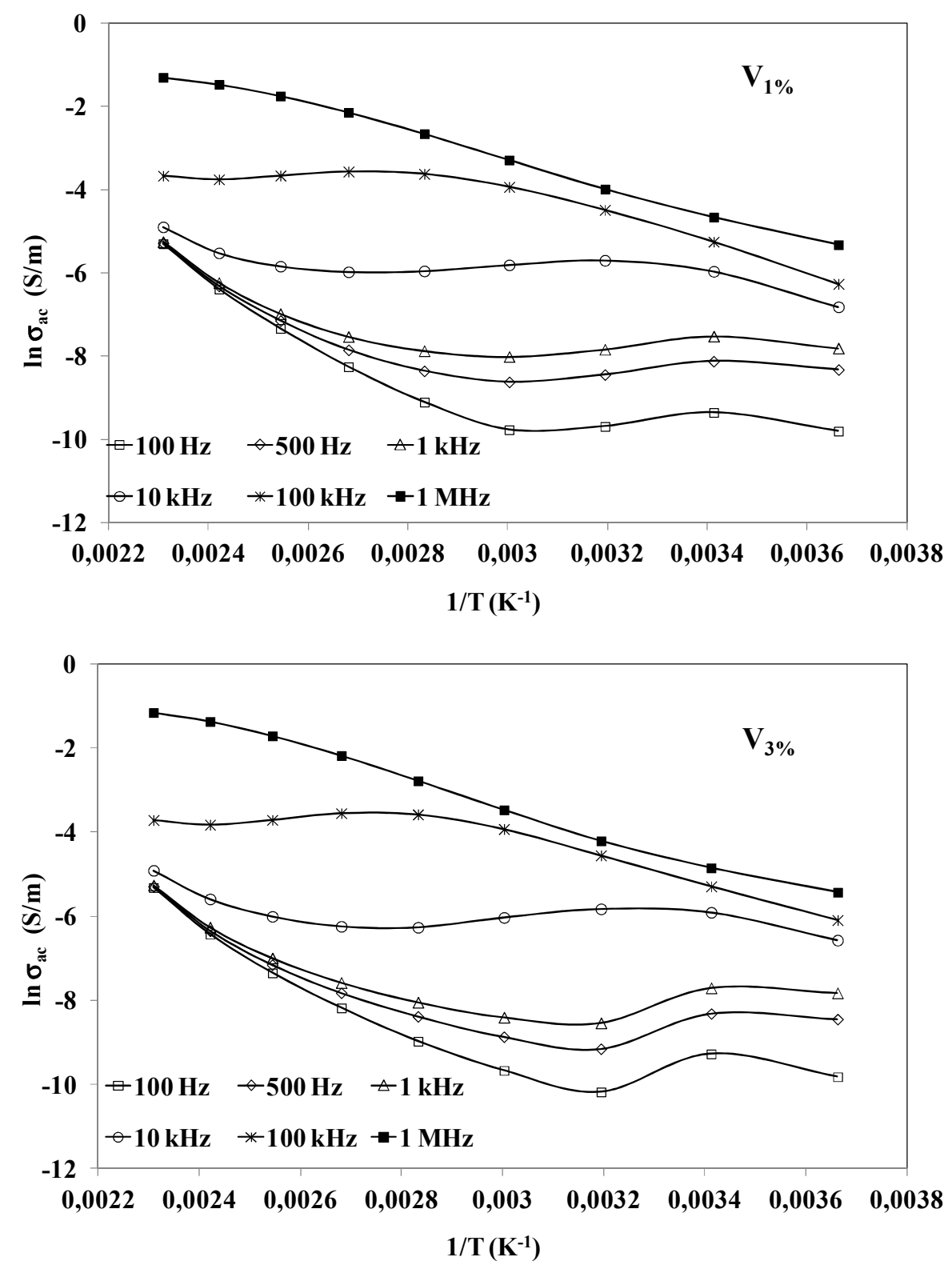

Figure 9. Arrhenius plot (ln $\sigma_{a c}$ as a function of 1/T) for pure and vanadium-doped CCTO samples.

Figure 9 shows the Arrhenius plots $\left(\ln \left(\sigma_{\mathrm{ac}}\right)\right.$ as a function of $1 / \mathrm{T})$ for un-doped and vanadium-doped CCTO at different frequencies. From this plot, according to Arrhenius principle, the value of activation energy can be deduced:

$$
\sigma_{\mathrm{ac}}=\sigma_{0} \exp \left(-\mathrm{E}_{\mathrm{a}} / \mathrm{k}_{\mathrm{B}} \mathrm{T}\right)
$$

where $\sigma_{0}$ is the pre-exponential factor, $E_{a}$ the activation energy, $\mathrm{k}_{\mathrm{B}}$ the Boltzmann constant $\left(8.61 \times 10^{-5} \mathrm{eV} \mathrm{K}^{-1}\right)$ and $\mathrm{T}$ the absolute temperature.

Real and imaginary parts of the dielectric constant of vanadium-doped samples are strongly temperature dependent, due to a relaxation process and the activation energies can be estimated by using the classical Arrhenius relation [23]:

$$
F_{p}(T)=v_{0} e^{-E a / k T}
$$

where $F_{p}(T)$ is the frequency of the peak of dielectric loss (Figure 7) at temperature $\mathrm{T}, v_{0}$ the pre-exponential factor or frequency factor, $E_{a}$ the activation energy of the relaxation processes obtained from straight inclination $\left(-E_{a} / \mathrm{k}_{\mathrm{B}}\right)$. From Figure 7 , the activation energies of the doped samples were calculated from Equation 4 and Figure 10 shows the plot of the relaxation frequency $\left(F_{p}\right)$ as a function of $1000 / \mathrm{T}$. Activation energy obtained for each doped compound was around $E_{a}=0.46 \mathrm{eV}$. Sinclar et al. [7, 8] found two types of resistances on bulk CCTO: the bulk $\left(R_{b}\right)$ and the grain boundary $\left(R_{g b}\right)$ with activation energies of $0.6 \mathrm{eV}$ and 0.08 $\mathrm{eV}$ respectively. The first one is close to the values found here. This suggests that the relaxation observed in our temperature and frequency ranges is related to the bulk resistance. Then the conductivity increases at high temperatures. 


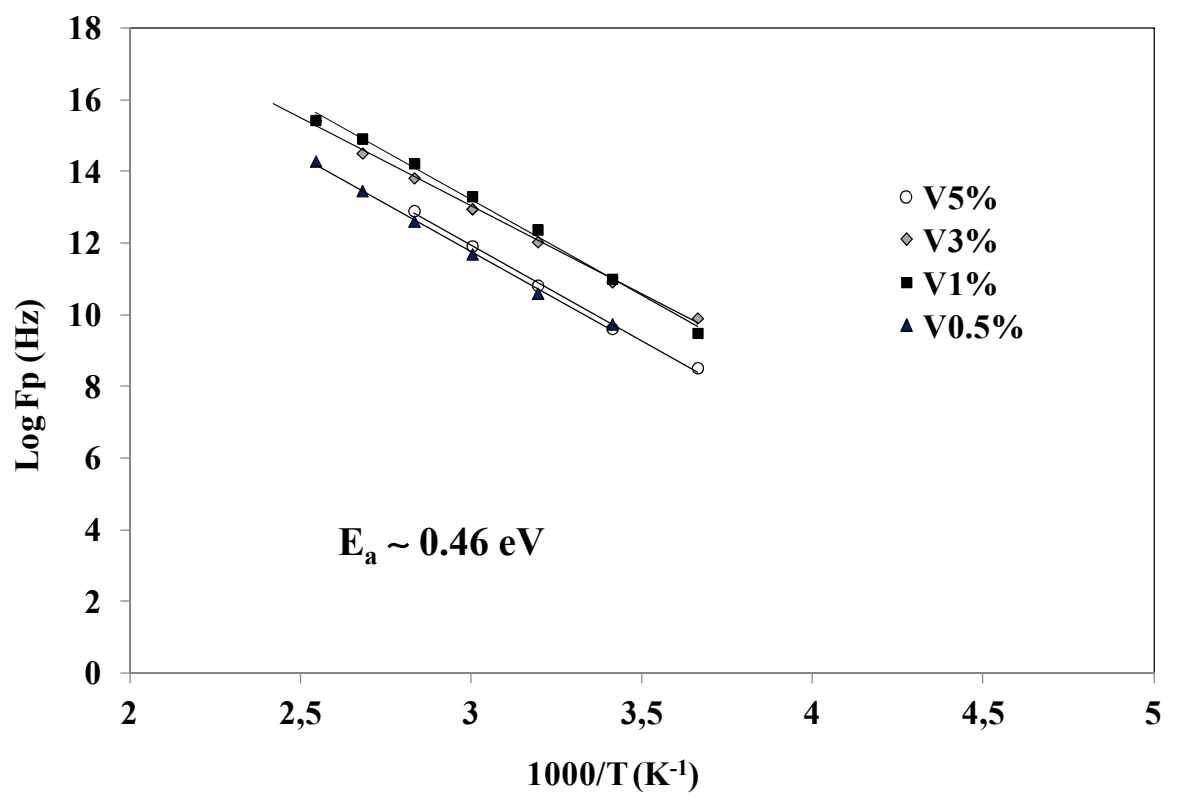

Figure 10. Activation energy of relaxation processes of vanadium-doped CCTO samples.

\section{Conclusion}

Vanadium doping of CCTO increases its lattice parameter and the grain size by partial substitution of titanium. At low frequency and room temperature, dielectric constants of undoped and doped CCTO are almost identical. In the presence of vanadium, above $1 \mathrm{kHz}$, it decreases due to a relaxation phenomenon that appears in our range of frequencies and temperatures. The relaxation peak moves towards lower temperatures when the vanadium content increases. AC conductivity has an effect at low frequency for doped samples while it only starts to play a role at high frequency for un-doped samples. The calculated activation energies were around $\boldsymbol{E}_{\boldsymbol{a}}=0.46 \mathrm{eV}$. This value is in fair agreement with values reported in the literature for bulk CCTO samples [7, 8] and CCTO film [17]. CCTO has been identified as an interesting material due to semiconducting grains and insulating grain boundaries [7]. Vanadium doping allows the relaxation to be shifted from very low frequencies to the $10-100 \mathrm{KHz}$ region, which leads to better dielectric properties at low frequencies (up to $1 \mathrm{kHz}$ ), but degrades them in the mid-frequency range.

\section{References}

[1] M. A. Subramanian, D. Li, N. Duan, B. A. Reisner, A. W. Sleight, "High dielectric constant in $A \mathrm{Cu}_{3} \mathrm{Ti}_{4} \mathrm{O}_{12}$ and $A \mathrm{Cu}_{3} \mathrm{Ti}_{3} \mathrm{FeO}_{12}$ phases," J. Solid State Chem., Vol. 151, pp. 323-325, 2000.

[2] W. D. Kingery, H. K. Bowen, D. R. Uhlmann, "Introduction to ceramics," second ed., John Wiley and Sons Inc., MA, pp. 913-974, 1976.

[3] K. Thomas Jacob, Chander Shekhar, Xiaogan Li, Girish M. Kale, "Gibbs energy of formation of $\mathrm{CaCu}_{3} \mathrm{Ti}_{4} \mathrm{O}_{12}$ and phase relations in the system $\mathrm{CaO}-\mathrm{CuO} / \mathrm{Cu}_{2} \mathrm{O}-\mathrm{TiO}_{2}$," Acta Mater., Vol. 56, pp. 4798-4803, 2008.

[4] A. K. Rai, K. D. Mandal, D. Kumar, O. Parkash, "Characterization of nickel doped CCTO: $\mathrm{CaCu}_{2.9} \mathrm{Ni}_{0.1} \mathrm{Ti}_{4} \mathrm{O}_{12}$ and $\mathrm{CaCu}_{3} \mathrm{Ti}_{3.9} \mathrm{Ni}_{0.1} \mathrm{O}_{12}$ synthesized by semi-wet route," J. Alloys Compd., Vol. 491, pp. 507-512, 2010.

[5] V. Brize, G. Gruener, J. Wolfman, K. Fatyeyeva, M. Tabellout, M. Gervais, F. Gervais, "Grain size effects on the dielectric constant of $\mathrm{CaCu}_{3} \mathrm{Ti}_{4} \mathrm{O}_{12}$ ceramics," Materials Science and Engineering, Vol. B 129, pp. 135-138, 2006.

[6] W. D. Kingery, H. K. Bowen, D. R. Uhlmann, "Introduction to Ceramics," second ed., John Wiley and Sons Inc., MA, pp. 913-974, 1976.

[7] D. C. Sinclair, T. B. Adams, F. D. Morrison, A. R. West, " $\mathrm{CaCu}_{3} \mathrm{Ti}_{4} \mathrm{O}_{12}$ : one-step internal barrier layer capacitor," Appl. Phys. Lett., Vol. 80, pp. 2153-2155, 2000.

[8] A. R. West, T. B. Adams, F. D. Morrison, D. C. Sinclair, "Novel high capacitance materials: $\mathrm{BaTiO}_{3}: \mathrm{La}$ and $\mathrm{CaCu}_{3} \mathrm{Ti}_{4} \mathrm{O}_{12}$," J. Eur. Ceram. Soc., Vol. 24, pp. 1439-1448, 2004.

[9] Virginie Brizé, Cécile Autret-Lambert, Jérôme Wolfman, Monique Gervais, Patrick Simon, François Gervais, "Temperature dependence of electron spin resonance in $\mathrm{CaCu}_{3} \mathrm{Ti}_{4} \mathrm{O}_{12}$ substituted with transition metal elements," sol. State sciences, Vol. 11, pp. 875-880, 2009.

[10] Pei Liu, Yuanming Lai, Yiming Zeng, Shuang Wu, Zihan Huang, Jiao Han, "Influence of sintering conditions on microstructure and electrical properties of $\mathrm{CaCu}_{3} \mathrm{Ti}_{4} \mathrm{O}_{12}$ (CCTO) ceramics, " J. of Alloys and Compounds, Vol. 650, pp. 59-64, 2015.

[11] Rosyaini Afindi Zaman, Mohamad Johari Abu, Julie Juliewatty Mohamed, Mohd Fadzil Ain, Zainal Arifin Ahmad, "Investigation of the Mixing Medium Effect on the Development of the CCTO Microstructure at $1000^{\circ} \mathrm{C}$." Procedia Chemistry, Vol. 16, pp. 906-909, 2016. 
[12] T. B. Adams, D. C. Sinclair, A. R. West, "Influence of Processing Conditions on the Electrical Properties of $\mathrm{CaCu}_{3} \mathrm{Ti}_{4} \mathrm{O}_{12}$ Ceramics," J. Am. Ceram. Soc., Vol. 89, pp. $3129,2006$.

[13] J. Li, A. W. Sleight, M. A. Subramanian, "Evidence for internal resistive barriers in a crystal of the giant dielectric constant material: $\mathrm{CaCu}_{3} \mathrm{Ti}_{4} \mathrm{O}_{12}$," Solid State Commun., Vol. 135, pp. 260, 2005.

[14] Juan Rodriguez-Carvajal, Laboratoire Léon Brillouin, (CEACNRS), CEA/Saclay, France.

[15] L. Fang, M. R. Shen, W. W. Cao, "Enhanced dielectric response of $\mathrm{GeO}_{2}$-doped $\mathrm{CaCu}_{3} \mathrm{Ti}_{4} \mathrm{O}_{12}$ ceramics," J. Appl. Phys., Vol. 95, pp. 6483, 2004.

[16] P. B. A. Fechine, A. F. L. Almeida, F. N. A. Freire, M. R. P. Santos, F. M. M. Pereira, R. Jimenez, J. Mendiola, A. S. B. Sombra, "Dielectric relaxation of $\mathrm{BaTiO}_{3}$ (BTO)$\mathrm{CaCu}_{3} \mathrm{Ti}_{4} \mathrm{O}_{12}$ (CCTO) composite screen-printed thick films at low temperatures," Mat. Chem. and Physics, Vol. 96, pp. 402408, 2006.

[17] Raman Kashayap, O. P. Thakur, R. P. Tandon, "Study of structural, diectric and electrical conduction behaviour of $\mathrm{Gd}$ substituted $\mathrm{CaCu}_{3} \mathrm{Ti}_{4} \mathrm{O}_{12}$ ceramics," ceramis international, vol. 38, pp. 3029-3037, 2012.
[18] L. F. Xu, P. B. Qi, X. P. Song, X. J. Luo, C. P. Yang, "Dielectric relaxation behaviors of pure $\mathrm{Pr}_{6} \mathrm{O}_{11}$-doped $\mathrm{CaCu}_{3} \mathrm{Ti}_{4} \mathrm{O}_{12}$ ceramics in high temperature range," J. of Alloys and Compounds, Vol. 509, pp. 7697-7701, 2011.

[19] B. S. Prakash, K. B. R. Varma, "Influence of sintering conditions and doping on the dielectric relaxation originating from the surface layer effects in $\mathrm{CaCu} / \mathrm{sub} 3 / \mathrm{Ti} / \mathrm{sub} 4 / \mathrm{O} / \mathrm{sub}$ 12/ ceramics," J. Phys. Chem. Solids, Vol. 68, pp. 490, 2007.

[20] X. J. Luo, C. P. Yang, S. S Chen, X. P. Song, H. Wang, K. Baerner, "The trap state relaxation related polarization in $\mathrm{CaCu}_{3} \mathrm{Ti}_{4} \mathrm{O}_{12}$," J. Appl. Phys., Vol. 108, pp. 1-5, 2010.

[21] A. M. M. Farea, Shalendra Kumar, Khalid Mujasam Batoo, Ali youssef, Alimuddin, "influence of frequency, temperature and composition on electrical properties of polycrystalline $\mathrm{Co}_{0.5} \mathrm{Cd}_{\mathrm{x}} \mathrm{Fe}_{2.5-\mathrm{x}} \mathrm{O}_{4}$ ferrites," Physica B, Vol. 403, pp. 684-701, 2008.

[22] W. Li, R. W. Schwartz, "AC conductivity relaxation processes in $\mathrm{CaCu}_{3} \mathrm{Ti}_{4} \mathrm{O}_{12}$ ceramics: grain boundary and domain boundary effects," Appl. Phys. Lett., Vol. 89, pp. 242906 (13), 2006.

[23] A. K. Jonscher, "Dielectric relaxation in solids," first ed., Chelsea Dielectrics Press, London, pp. 62-115, 1983. 